\title{
Design and Control of SLIDER: An Ultra-lightweight, Knee-less, Low-cost Bipedal Walking Robot
}

\author{
Ke Wang ${ }^{1}$, David Marsh ${ }^{2}$, Roni Permana Saputra ${ }^{1,3}$, Digby Chappell ${ }^{1}$, \\ Zhonghe Jiang ${ }^{4}$, Bethany Kon ${ }^{5}$, and Petar Kormushev ${ }^{1}$
}

\begin{abstract}
Most state-of-the-art bipedal robots are designed to be highly anthropomorphic and therefore possess legs with knees. Whilst this facilitates more human-like locomotion, there are implementation issues that make walking with straight or near-straight legs difficult. Most bipedal robots have to move with a constant bend in the legs to avoid singularities at the knee joints, and to keep the centre of mass at a constant height for control purposes. Furthermore, having a knee on the leg increases the design complexity as well as the weight of the leg, hindering the robot's performance in agile behaviours such as running and jumping.

We present SLIDER, an ultra-lightweight, low-cost bipedal walking robot with a novel knee-less leg design. This nonanthropomorphic straight-legged design reduces the weight of the legs significantly whilst keeping the same functionality as anthropomorphic legs. Simulation results show that SLIDER's low-inertia legs contribute to less vertical motion in the center of mass (CoM) than anthropomorphic robots during walking, indicating that SLIDER's model is closer to the widely used Inverted Pendulum (IP) model. Finally, stable walking on flat terrain is demonstrated both in simulation and in the physical world, and feedback control is implemented to address challenges with the physical robot.
\end{abstract}

\section{INTRODUCTION}

With very few exceptions, most bipedal robots have knees. Whilst robots with knees are more anthropomorphic, nearly all implementations exhibit an artificial, crouched walking gait with bent knees, to accommodate the Linear Inverted Pendulum Model's assumption of a fixed height of the center of mass [1]. This leads to high power consumption at the knee. Even with this bend, walking gates require operation very close to singularities in the Jacobian when solving inverse kinematics (IK) or inverse dynamics [2]. Furthermore, knees add design complexity and weight. Heavier legs add burden on actuators and hinder the robot's capability to make fast movements, which is commonly required in running, jumping, hopping, and disturbance rejection. A

\footnotetext{
${ }^{1}$ Ke Wang, Roni Permana Saputra, Digby Chappell and Petar Kormushev are with the Robot Intelligence Lab, Dyson School of Design Engineering, Imperial College London, UK

${ }^{2}$ David Marsh is with the Department of Mechanical Engineering, Imperial College London, UK

${ }^{3}$ Roni Permana Saputra is also with the Research Center for Electrical Power and Mechatronics, Indonesian Institute of Sciences - LIPI, Indonesia

${ }^{4}$ Zhonghe Jiang is with the Department of Electrical and Electronic Engineering, Imperial College London, UK

${ }^{5}$ Bethany Kon is with the Department of Bioengineering, Imperial College London, UK

\{k.wang17, david.marsh18, zhonghe.jiang18, r.saputra16, d.chappel119, bethany.kon19, p.kormushev\} @imperial.ac.uk
}

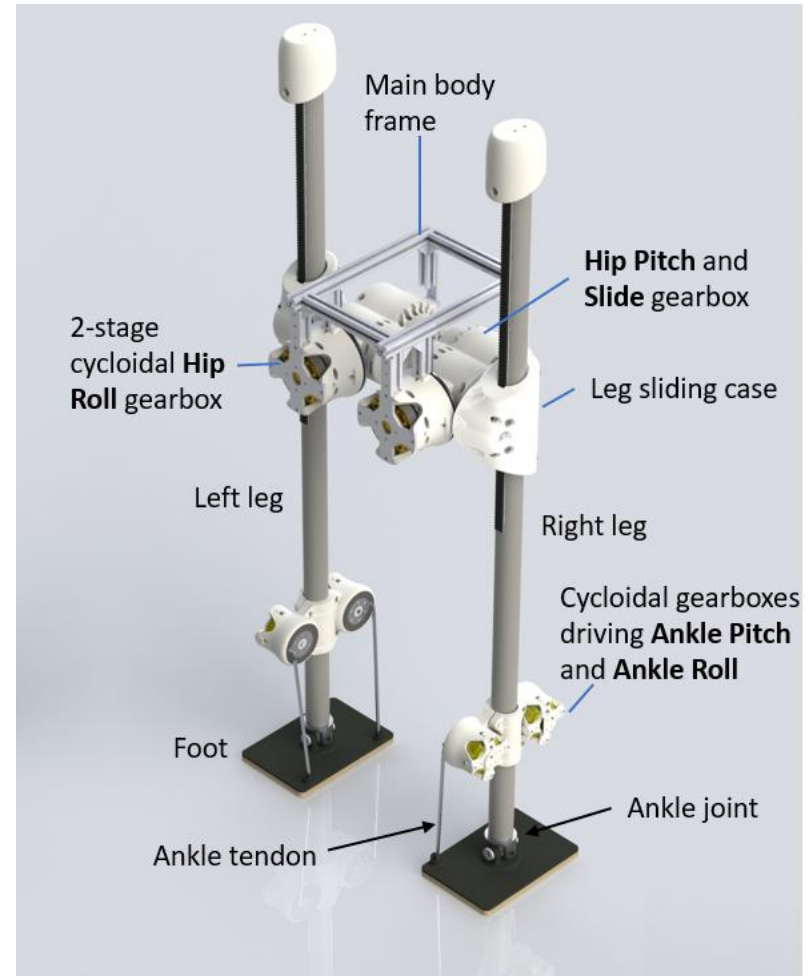

Fig. 1: A model of the SLIDER robot. It has $10 \mathrm{DoF}$ and straight legs made of carbon fiber reinforced polymer. It is designed to be ultra-lightweight and suitable for agile locomotion.

detailed comparison between the anthropomorphic and the straight-legged design can be seen in [3].

There are a few examples of robots that have legs without knees. Raibert and his colleagues [4] demonstrated a somersaulting 3D biped robot which had pneumatic telescoping legs. Kajita et al. [5] showed 3D walking of the $12 \mathrm{DoF}$ telescoping bipedal robot Meltran V. In [6] is a video demonstrating a knee-less bipedal robot by Schaft, but the design details are unpublished. In [3], the concept of the knee-less SLIDER robot is originally introduced, along with a control strategy, but only simulation results are shown. SLIDER's novelty comes from its leg sliding mechanism similar to a servo belt, which is better than telescoping legs at producing lightweight, inexpensive agility.

Although bipedal robot technology has advanced significantly [7][8][9], only a few labs and research institutions are capable of making or purchasing a bipedal robot of 
similar size to a human. Recently there has been a big progress in building low-cost quadruped robots using the modular motor which has a build-in one-stage planetary gear set [10]. However, this kind of motor hasn't reached the performance to provide enough torque required for the hip joints of bipedal robots. On SLIDER, nearly all parts are manufactured in-house and most parts are 3D-printed, making SLIDER lightweight and low-cost. To achieve this, we used cycloidal gearboxes, which are easier to manufacture in-house compared with harmonic drives. SLIDER is built with the spirit to provide a low-cost research platform for validating and experimenting novel bipedal walking control algorithms.

In this paper, we present the mechanical design, electronics and control strategy of SLIDER, an ultra-lightweight, kneeless, low-cost bipedal walking robot. The comparison of the CoM motion between SLIDER and an antropomorphic bipedal walking robot is presented, showing that SLIDER's low-inertia leg has less effect on the vertical motion of CoM. We validate the design of SLIDER by demonstrating walking on flat terrains both in simulation and on the physical robot.

This paper is organized as follows: Section 2 describes the mechanical design of SLIDER and Section 3 briefly outlines the electronics and software architecture. In Section 4 , we introduce the control strategy that enables SLIDER to walk and Section 5 describes and analyzes the experimental results. Finally, in Section 6, we present relevant future work for SLIDER.

\section{DESIGN OVERVIEW}

Figure 1 shows an illustration of the SLIDER robot; a knee-less bipedal walking robot design comprising of a pair of feet, legs, and main body frame. This robot has 10 joints with 5 degrees of freedom (DoFs) at each leg and has been designed to be ultra-lightweight and low-cost, but suitable as a bipedal locomotion research platform.

SLIDER's most distinctive feature, its prismatic "slide" leg articulation, imposes both inherent benefits and constraints onto its design when compared to more traditional humanoid robots. Its most notable benefit is the mass and leg complexity reduction attained because there is no knee. However, the hip is burdened with another actuator, complicating the design. To take advantage of the benefits of a knee-less design (lower leg mass) and better approximate an inverted pendulum model, SLIDER's primary design goals are: 1) lightweight and compact joints; 2) concentration of mass as close to main body frame as possible; 3) low development cost and 4) easily modifiable design, to allow the rapid addition of new sensors and mechanical improvements.

\section{A. SLIDER's Design vs Anthropomorphic Design}

1) Mass Distribution: The conventional anthropomorphic leg design has a 3-1-2 DoF distribution (3 DoF in hip, $1 \mathrm{DoF}$ in knee, 2 DoF in ankle). SLIDER's non-anthropomorphic design has a 4-0-2 DoF distribution, with 4 DoF in the hip (hip roll, hip pitch, hip yaw, and a prismatic "slide"), and

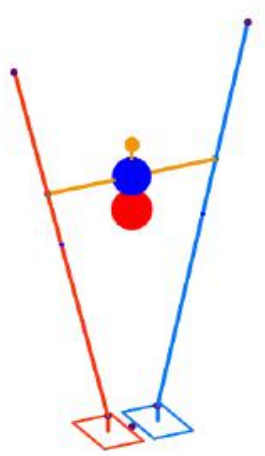

(a) SLIDER

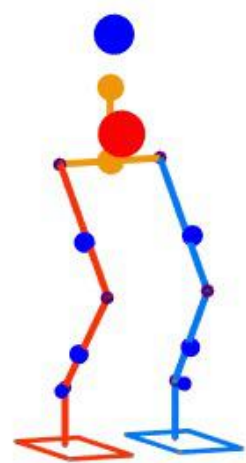

(b) COMAN
Fig. 2: Illustration of SLIDER's mass distribution in comparison to an anthropomorphic bipedal robot, COMAN. The size of spheres correspond to the mass at that specific links. The red sphere represents the CoM position.

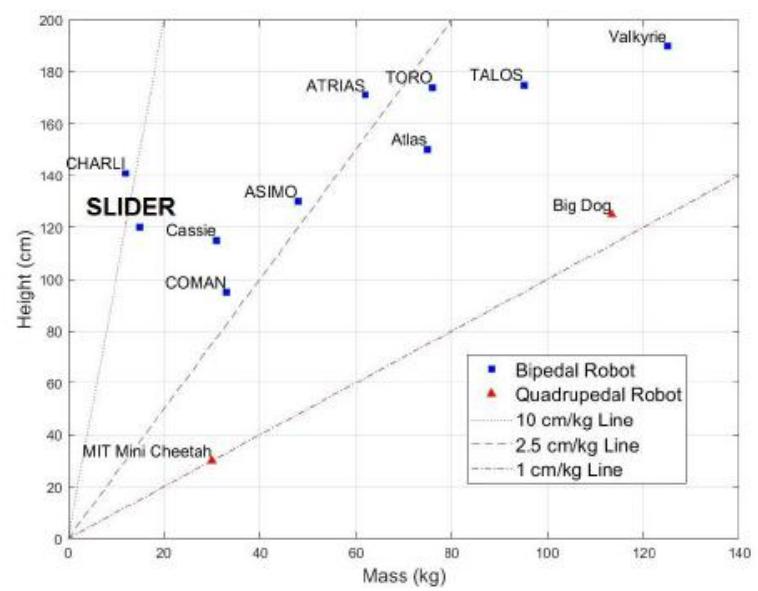

Fig. 3: Height-mass relationship of selected robots.

no knee. ${ }^{1}$ This significantly changes the mass distribution of SLIDER, making the legs lighter, and shifting the CoM higher. SLIDER's mass (using off-board computer and power supply) and height are $15.0 \mathrm{~kg}$ and $1.2 \mathrm{~m}$, respectively.

Figure 2 visualises the comparison of the mass distribution between SLIDER and COMAN robot (an anthropomorphic bipedal robot) [11]. It can be seen from the figure that, in COMAN, a considerable amount of the weight is distributed at each leg and also at its pelvis. Meanwhile, in SLIDER robot design, almost all the weight is concentrated at the main frame while a small amount of weight is distributed at the robot legs. This mass distribution is one of the most distinctive features of SLIDER robot design, in comparison to the conventional anthropomorphic designs.

Table I presents the mass distribution at each link of SLIDER and COMAN. The table highlights SLIDER's lightweight leg design, showing that we have achieved one

\footnotetext{
${ }^{1}$ Please note that the hip yaw DoF is not yet implemented in the current version of SLIDER.
} 


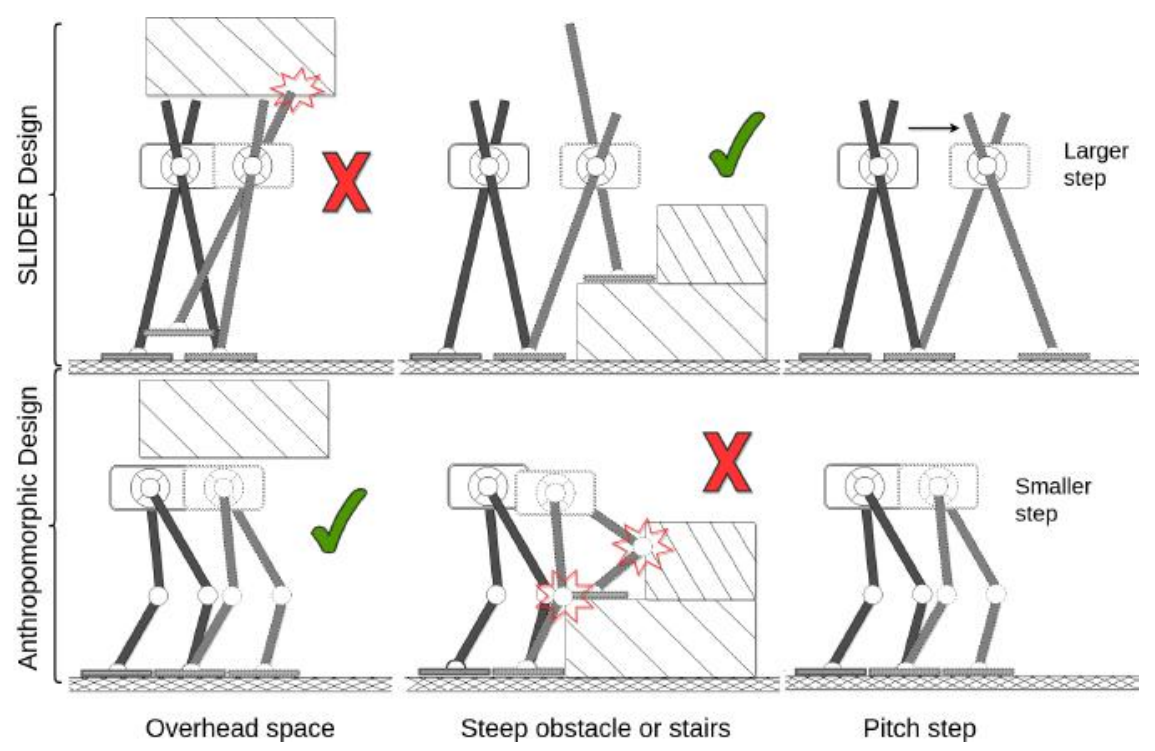

Fig. 4: SLIDER's Design vs Anthropomorphic Design.

TABLE I: Overview of SLIDER and COMAN's mass distribution

\begin{tabular}{|c|c|c|c|c|c|c|}
\hline & Pelvis & Hip & Upper Leg & Lower Leg & Ankle & Foot \\
\hline SLIDER* & - & $5.8 \mathrm{Kg}^{* *}$ & \multicolumn{2}{|c|}{$0.6 \mathrm{Kg}$} & $1.2 \mathrm{Kg}$ & $0.4 \mathrm{Kg}$ \\
\hline COMAN* & $5.5 \mathrm{Kg}$ & $1.2 \mathrm{Kg}$ & $1.7 \mathrm{Kg}$ & $1.4 \mathrm{Kg}$ & $0.7 \mathrm{Kg}$ & $0.9 \mathrm{Kg}$ \\
\hline
\end{tabular}

* Each mass given is for one leg only.

** The mass of the leg sliding case is included in the mass of the hip.

of our objectives; ultra-lightweight bipedal robot design. Further investigation of the weight comparison between SLIDER and other existing legged robots, Figure 3 also highlights the fact that SLIDER robot inherit ultra-lightweight design. Compared to other legged robots reported in the literature, SLIDER's height-mass ratio is one of the highest ratio by approximately $6.67[\mathrm{~cm} / \mathrm{kg}]$.

2) Workspace Comparison: Regarding workspaces, SLIDER benefits from its lack of knees in that it can navigate large obstacles or steep stairs, and larger steps are significantly easier to achieve. However, the prismatic leg means that clearance above the robot is required, meaning overhead obstacles are unsuitable for navigation (see Figure 4). In relation to potential applications, this makes SLIDER well suited to agile locomotion and walking on uneven terrains.

\section{B. SLIDER Mechanical Design Overview}

Figure 5 illustrates the kinematic diagram of SLIDER robot. It can be seen that each leg comprising of five joints, (i) hip roll, (ii) hip pitch, (iii) hip slide, (iv) ankle pitch, and (v) ankle roll. The specification of these joints are provided in Table II. As the prismatic "slide" leg articulation is the most distinctive feature of SLIDER robot, an overview of this mechanism will be explained in the following subsection. Moreover, the cycloidal gear system as one of the main parts of SLIDER mechanical design to achieve compact, lightweight and low-cost design of SLIDER robot will be
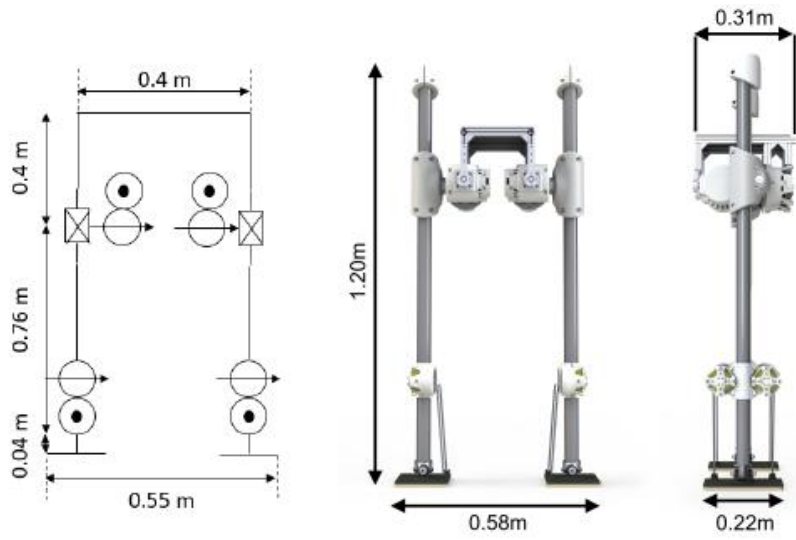

Fig. 5: Joint configuration of SLIDER (left) and physical SLIDER (right)

TABLE II: Overview of SLIDER's joint specifications

\begin{tabular}{|c|c|c|c|c|}
\hline Joint & Motor & $\begin{array}{c}\text { Gear } \\
\text { Ratio }\end{array}$ & $\begin{array}{c}\text { Gear } \\
\text { Arrangement }\end{array}$ & Range \\
\hline $\begin{array}{c}\text { Hip } \\
\text { Roll }\end{array}$ & $\begin{array}{c}\text { Multistar } \\
9225-160 \mathrm{Kv}\end{array}$ & $36: 1$ & $\begin{array}{c}\text { 2 stage cycloidal } \\
\text { reduction }\end{array}$ & $\begin{array}{c} \pm 30^{\circ} \text { to } \\
\mp 90^{\circ}\end{array}$ \\
\hline $\begin{array}{c}\text { Hip } \\
\text { Pitch }\end{array}$ & $\begin{array}{c}\text { Multistar elite } \\
5010-274 \mathrm{Kv}\end{array}$ & $38.06: 1$ & $\begin{array}{c}\text { 2 stage epicyclic } \\
\text { + 1 stage spur }\end{array}$ & $\pm 180^{\circ}$ \\
\hline $\begin{array}{c}\text { Hip } \\
\text { Slide }\end{array}$ & $\begin{array}{c}\text { Multistar elite } \\
5010-274 \mathrm{Kv}\end{array}$ & $\begin{array}{c}1.23 \\
\mathrm{~cm} / \mathrm{rev}\end{array}$ & $\begin{array}{c}\text { 1 stage epicyclic+ } \\
\text { 1 stage herringbone } \\
\text { +rack and pinion* }\end{array}$ & $\begin{array}{c}-30 \text { to } \\
+40 \mathrm{~cm}\end{array}$ \\
\hline $\begin{array}{c}\text { Ankle } \\
\text { Pitch }\end{array}$ & $\begin{array}{c}\text { Quanum MT } \\
5208-335 \mathrm{Kv}\end{array}$ & $15: 1$ & $\begin{array}{c}\text { 1 stage cycloidal } \\
\text { reduction }\end{array}$ & $\pm 30^{\circ}$ \\
\hline $\begin{array}{c}\text { Ankle } \\
\text { Roll }\end{array}$ & $\begin{array}{c}\text { Quanum MT } \\
5208-335 \mathrm{Kv}\end{array}$ & $15: 1$ & $\begin{array}{c}\text { 1 stage cycloidal } \\
\text { reduction }\end{array}$ & $\pm 21^{\circ}$ \\
\hline
\end{tabular}

* One-stage herringbone and rack and pinion arrangements are within the leg sliding case. 
TABLE III: Comparison of ServoBelt drive mechanism with other conventional prismatic joint transmission systems

\begin{tabular}{|l|c|c|c|c|}
\hline Prismatic Joint Transmission & Light-weight & Low cost & Precission & Maximum Load \\
\hline Lead screw & No & No & High & High \\
\hline Ball screw & No & No & High & Medium \\
\hline Rack and pinion drive & No & Medium & Medium (depends on gear backlash) & Medium \\
\hline Conventional belt and pinion drive & Yes & Yes & Medium (depends on belt tension) & High \\
\hline ServoBelt linear drive & Yes & Yes & High \\
\hline
\end{tabular}

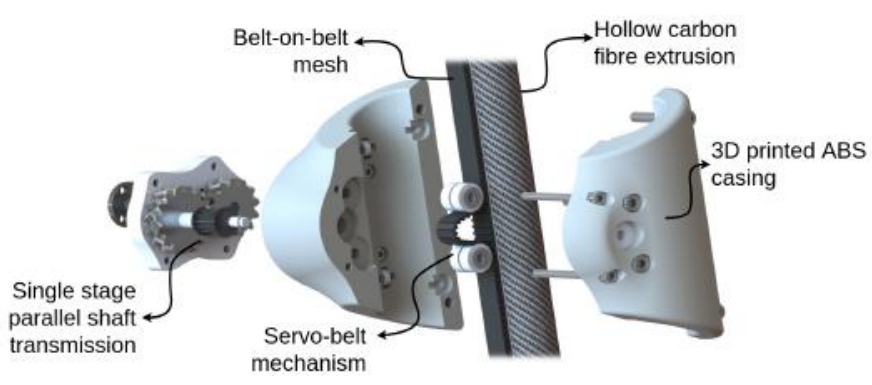

(a) The exploded view of SLIDER's slide mechanism assembly.

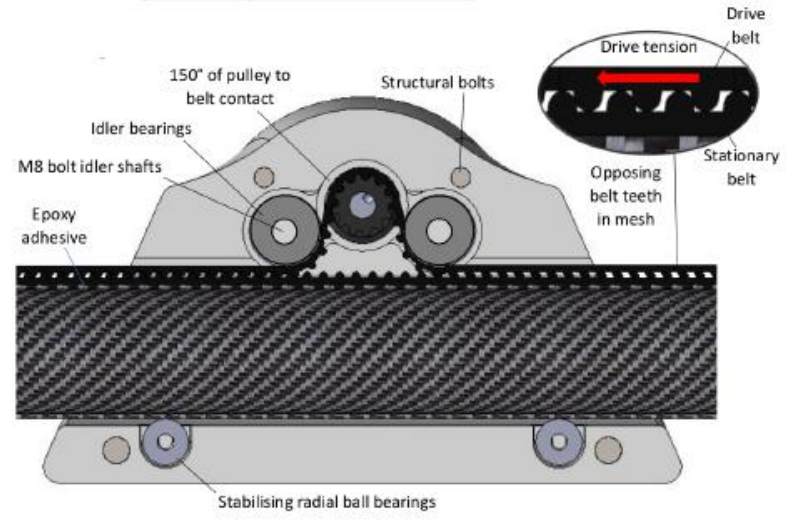

(b) The illustration of the ServoBelt mechanism for slide joint linear motion.

Fig. 6: SLIDER's slide joint design comprising of a two layer sandwiched belt system with a fixed and free belt, and a rotating timing-belt pulley.

also presented in the consecutive subsection.

1) Leg Sliding Joint using ServoBelt Drive Mechanism:

To realise the leg slide mechanism in SLIDER, the translation of rotary motion from the joint actuator (i.e. controlled motor) to linear motion of the leg is required. As maintaining an ultra-lightweight design is one of the main goals, this slide leg mechanism design needs to be: (i) compact, (ii) lightweight, (iii) reliable, and have (iv) strong linear actuation. To achieve these, we propose to implement the ServoBelt mechanism comprising of a two layer sandwiched belt system with a fixed and free belt, and a rotating timingbelt pulley. This mechanism is exclusively introduced by Bell-Everman in [12]. The ServoBelt mechanism is capable of an increased load capability, reduced backlash, and a high actuation speed. As well as providing a strong and high-precision transmission, the proposed design ensures the compact, light-wight and reliable mechanism. Table III presents the comparison of the ServoBelt drive mechanism

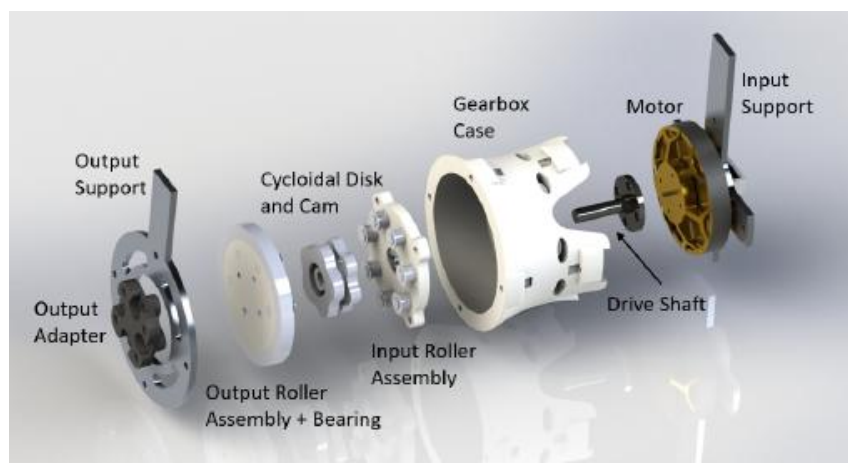

Fig. 7: The design of SLIDER's hip-roll joint using a double-stage cycloid drive transmission. The cycloid drive is designed to achieve a large transmission ratio (36:1) while maintaining the compact design structure.

with other conventional prismatic joint transmission systems.

To implement the ServoBelt mechanism for SLIDER's slide joint, we use a pair of heavy duty timing belts: a drive belt and a stationary belt. The stationary belt is fixed to the carbon fibre leg, while the drive belt contacts with the timingbelt pulley connected to the output-shaft of the motor-drive gearbox. Figure 6a demonstrates the assembly of the main components of the servo-belt mechanism and the illustration of the transmission mechanism is presented in Figure 6b.

2) Cycloidal Gears for Low-cost and Lightweight Transmission Gearbox: One of the most distinctive features of the design of SLIDER is the use of cycloidal gears for gearbox transmission instead of using a harmonic drive, which is widely used for joint transmission in many anthropomorphic bipedal robot designs. Several studies have been introduced in the evaluation and comparison of harmonic drive and cycloidal gear in application to robotic transmissions, including studies in [13] and [14]. According to the study presented by Sensinger and Lipsey in [13], it is found that cycloid drive could be considered for robotic transmissions, especially for the applications where a compact design and efficiency are valued above precision (i.e. low backlash), including the ratio ripple considerations.

In relation to our SLIDER robot design, we believe that adopting cycloid drive transmission would be a legitimate design selection. The main reasons behind this design selection are (i) to achieve high ratio transmission with a compact design, (ii) to accelerate design iteration by leveraging the rapid prototyping using additive manufacturing, (iii) to achieve low-cost and light-weight design by compromising sufficient precision in comparison to harmonic drive, and (iv) 
we believe that by implementing a suitable control strategy, we could cope with the precision issue as it will be confirmed in the experiments. We adopt this cycloidal gearbox design for the hip roll joint transmissions and ankle hip and ankle roll joint transmission. Figure 7 shows SLIDER's hip-roll joint that uses a double-stage cycloid drive transmission.

\section{ELECTRONICS AND SOFTWARE}

\section{A. Sensors and Actuators}

All SLIDER joints are driven by brushless DC motors, specifications of the motors of each joint can be found in Table II. Motors are controlled by ODrives [15] (open-source high performance motor controllers which can handle peak currents of more than 100A per motor). Each ODrive is capable of controlling 2 motors. ODrives have a cascaded style motor controller with position, velocity and current control loop, all updated with $8 \mathrm{kHz}$. A programmable interface through Python is provided to the user.Each joint has an AS5047P absolute encoder on the motor side, connected to the ODrive. To provide feedback signals on the output side, 3 Bosch BNO055 IMUs are mounted on the robot. One IMU is mounted on the base frame to measure the orientation of the base, with the other two IMUs mounted on the left and right leg to measure the pitch and roll angle of each leg with respect to the ground. Each foot has 4 load cells mounted on the bottom to provide the Center of Pressure (CoP) with $125 \mathrm{~Hz}$.

\section{B. Software}

We use Robot Operating System (ROS) to reduce the computational load of the main controller program. Local processing of ODrives, IMUs, load cells is handled by separate ROS nodes and the communication with the main controller is handled by ROS messages. This allows the additional functionality to be easily added. The code is written in Python, which is fast enough for now. We use Gazebo as our simulation environment. Gazebo is deeply integrated with ROS and ros_control is used for the low-level joint control. Gazebo uses ODE as its default physics engine. Although ODE has worse reliability than the OpenHRP/AIST dynamics engine, as stated in [16], we set the max step in ODE to be 0.0005 so the reliability is guaranteed for simulating walking.

\section{CONTROLLER DESIGN}

Because SLIDER has light-weight straight legs, Linear Inverted Pendulum model (LIPM) can be used for generating flat terrain walking motion. The inverse kinematics is adjusted for SLIDER's special design. To make physical SLIDER walk, trajectory compensation and feedback control are necessary, which will be discussed in detail at next section.

\section{A. Walking Pattern Generator}

The walking gait is generated offline by a two-stage dynamically consistent gait pattern generator (see Fig. 8) [17]. The first stage accepts the reference CoM velocity as the input and uses the simplified LIP model based on Eq. 1 to generate the CoM and feet reference trajectories. The second stage contains a preview controller with a dynamical filter included. The dynamic filter uses multibody dynamics to make the generated gait more dynamically feasible for the real robot. It uses the reference trajectories of CoM and feet from the first stage to generate the final walking pattern.

$$
\begin{aligned}
& \mathrm{ZMP}_{x}^{\text {ref }}=x-\frac{z}{g} \ddot{x}, \\
& \mathrm{ZMP}_{y}^{r e f}=y-\frac{z}{g} \ddot{y} .
\end{aligned}
$$

\section{B. Inverse Kinematics}

Since SLIDER has knee-less legs, the inverse kinematics result in a simple geometric problem that can be easily solved by an analytical method [18]. The method assumes that the three hip joint axes intersect each other at one point, which is not true for SLIDER because the axis of hip roll does not intersect the leg (see Fig. 5). To compensate this difference, we implement the following slide joint compensation:

$$
\tilde{q}_{3}=q_{3}-\alpha L \tan q_{3}
$$

where $q_{3}$ is the original value of hip slide, $\alpha$ is a coefficient determining the compensation multiplier of the original trajectory and is chosen to be 0.5 for our implementation. $L$ is the distance between the hip roll axis and the leg in the $y$ direction.
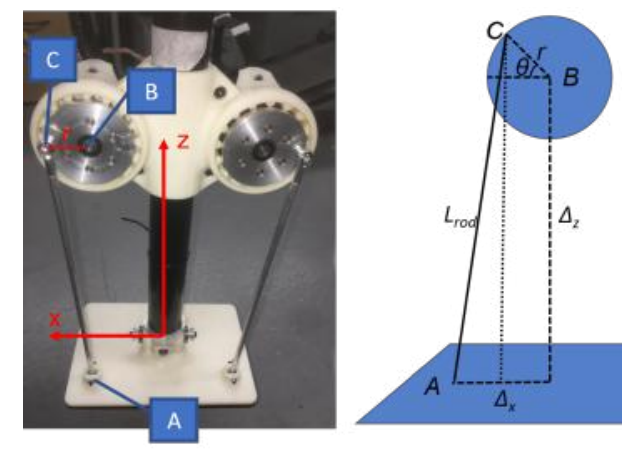

Fig. 9: Illustration of the inverse kinematics for the ankle.

The ankle pitch and roll motions are coupled by a six bar linkage structure, a geometric method is proposed to solve this Inverse Kinematics (IK) problem. As seen in Fig. 9, the front point $A$ of the right foot is driven by the motor centered at $B$. A base frame $\Sigma_{L}$ is attached to the leg, with the origin located at the intersection of the two ankle axes with the $\mathrm{Z}$-axis pointing upward and $\mathrm{x}$-axis pointing forward. A local frame $\Sigma_{F}$ is attached to the foot with the same origin as $\Sigma_{L}$. We assume both $A$ and $B$ is fixed in local frame $\Sigma_{L}$ and are expressed as ${ }^{F} p_{A}$ and ${ }^{L} p_{B}$ respectively. Given the desired ankle roll angle $q_{4}$ and ankle pitch angle $q_{5}$, the position of $A$ expressed in the base frame can be calculated as follows:

$$
{ }^{L} p_{A}=R_{x}\left(q_{4}\right) R_{y}\left(q_{5}\right)^{F} p_{A},
$$

where $R_{x}, R_{y}$ are the rotation matrices with respect to the $x$ and $y$ axes. We can then establish a relationship for the 


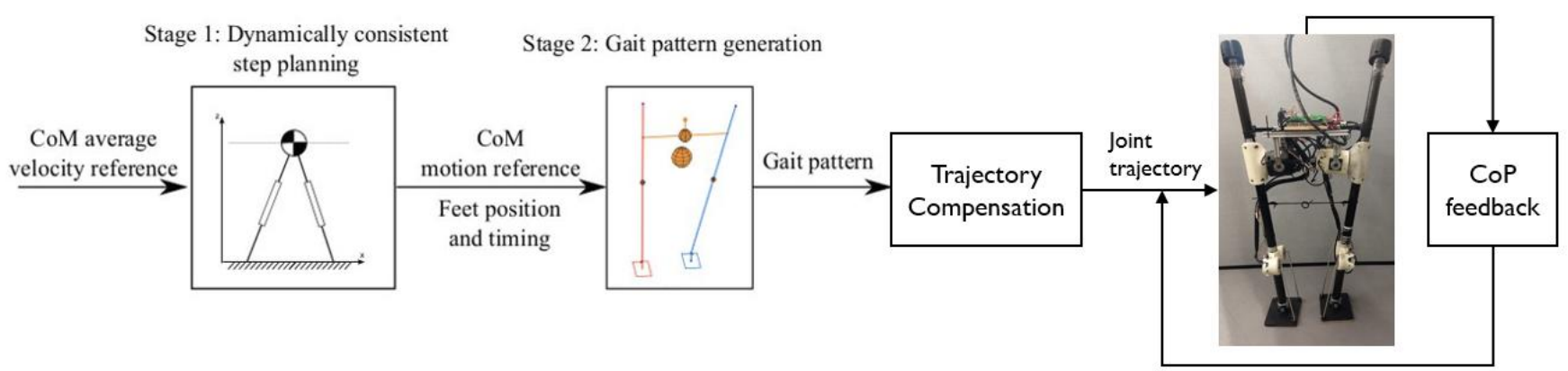

Fig. 8: The control diagram of SLIDER.

angle of rotation of the motor $\theta$ as denoted in Fig. 9:

$$
\left(r \cos \theta-\Delta_{x}\right)^{2}+\left(r \sin \theta+\Delta_{z}\right)^{2}=L_{\text {rod }}^{2},
$$

where $\left[\begin{array}{ccc}\Delta_{x} & \Delta_{y} & \Delta_{z}\end{array}\right]$ is the absolute value of each individual element of $\left({ }^{L} p_{A}-{ }^{L} p_{B}\right)^{T}, r$ is the radius of the motor output plate and $L_{\text {rod }}$ is the length of the rod. This equation can be solved symbolically by Symbolic Math Toolbox in MATLAB and the two solutions are shown below:

$$
\begin{aligned}
& \theta_{1}=-\log \left(\frac{\Delta_{x}^{2}+\Delta_{z}^{2}+r^{2}-L_{\text {rod }}^{2}+s}{2\left(\Delta_{x} r+\Delta_{z} r i\right)}\right) i, \\
& \theta_{2}=-\log \left(\frac{\Delta_{x}^{2}+\Delta_{z}^{2}+r^{2}-L_{r o d}^{2}-s}{2\left(\Delta_{x} r+\Delta_{z} r i\right)}\right) i,
\end{aligned}
$$

with

$$
\begin{aligned}
s= & \sqrt{-\left(-\Delta_{x}^{2}-\Delta_{z}^{2}+r^{2}+2 r L_{\text {rod }}+L_{\text {rod }}^{2}\right)} \\
& \times \sqrt{\left(\Delta_{x}^{2}+\Delta_{z}^{2}-r^{2}+2 r L_{\text {rod }}-L_{\text {rod }}^{2}\right)} .
\end{aligned}
$$

The existence of two solutions is expected because the circle centered at $A$ with radius $L_{\text {rod }}$ has two intersections with the motor output plate. We choose the solution in $[-\pi, \pi]$ to restrict the motion of the motor. The result can also help us determine whether a gait is executable or not, because a complex result means that the desired ankle pitch and roll motion is infeasible.

\section{EXPERIMENTS}

\section{A. Simulation}

SLIDER and COMAN were both simulated walking with identical gait pattern generation and control strategies. Figure 11 shows that SLIDER's centre of mass moves less - it has both reduced sway, and remains closer to being at a constant height.

Both a Gazebo simulation of SLIDER and the physical robot were tested in flat terrain walking trials. The Gazebo version possesses the same kinematic and dynamic properties as the real robot. Four contact sensors are mounted at the bottom of each foot. A trajectory is generated offline in MATLAB with the sliding joint compensation 2, then the trajectory is tracked in simulation using a PD controller. In simulation, SLIDER can walk very stably at a speed of 0.5 m/s, see Fig 10 .

\section{B. Physical Experiments}

The physical SLIDER uses a trajectory tracked at a frequency of $500 \mathrm{~Hz}$. Due to the backlash and deformation of hip pitch and hip roll joints, tracking offline generated trajectory doesn't result in stable walking. To achieve stable walking on the physical SLIDER, both feedforward compensation and feedback controller for stablization are used. For feedforward compensation, three compensations were added to the offline generated trajectory to help counteract various mechanical imperfections:

1) Linear Offset - Constant offsets were added to precisely transit from the calibrated home position (orthogonal axes at each joint with slide at a reference location) to the neutral position (static, balanced standing, which serves as the starting position for walking gaits). The offsets can be adjusted to account for imprecise calibration of the home position.

2) Non-Scheduled Scaling - Because of the lightweight construction, SLIDER has large loaded deformations in its joints, which introduces error between the requested and actual positions during movements. To adjust for these errors, the commanded changes in position can be linearly scaled. The hip pitch joints make most use of this compensation to counteract the particularly high deformation present.

3) Scheduled Scaling - To allow scaling of commanded position changes during only certain portions of the walking gait, scheduled scaling is used. Due to loaded deformation which almost exclusively occurs during single support phase, the commanded roll positions of the support leg are scaled during single support phase only.

During early walking tests, non-flat foot impacts with the floor often caused loss of balance. It was determined that a control strategy would need to be applied, utilizing the real data about the CoP. There is also an observation that due to the high CoM position of SLIDER, a small disturbance on ankle can cause the fall of the robot. Load cells are used to measure the CoP and calculate the actual ZMP in single and double support phase, based on the algorithm proposed by [19]. A PD feedback control law is used to track the desired ZMP in both phases. 

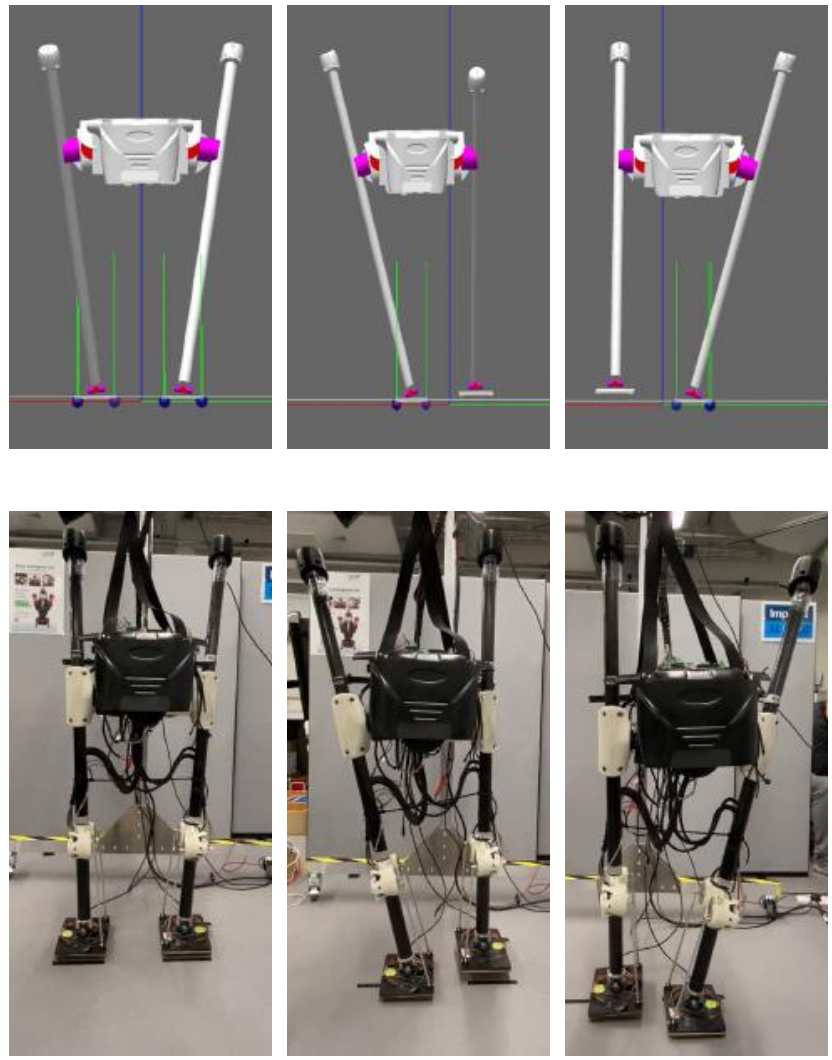

Fig. 10: The top 3 snapshots show SLIDER walking in simulation, with green vectors indicating the ground reaction forces. From left to right: double support phase, single support phase with right leg as support leg, single support phase with left leg as support leg. The bottom 3 photos show the physical SLIDER robot walking on flat floor. From left to right: same order as the top images.

By applying a PD control law, the angles of the ankle joints are adjusted online, even when the leg positions are not fully tracked precisely. With a combination of offline trajectory generation and online CoP feedback control, SLIDER walks with a speed of $0.10 \mathrm{~m} / \mathrm{s}$, see Fig 10 .

\section{DISCUSSION}

From Figure 11, SLIDER's centre of mass remains closer to a constant height; this means that the linear inverted pendulum model used to plan steps and generate a reference CoM motion is more valid than the anthropomorphic walking robot, COMAN.

The physical robot differs from simulation in one key area: backlash in the physical robot is large. As mentioned in II-B.2, cycloidal gears are useful when compactness and efficiency are more important than precision. In the physical robot, this leads to significant backlash occurring in the hip roll joints, meaning stable walking without control feedback (open loop) is challenging. Adding control feedback by measuring the centre of pressure of each foot greatly improves stability.
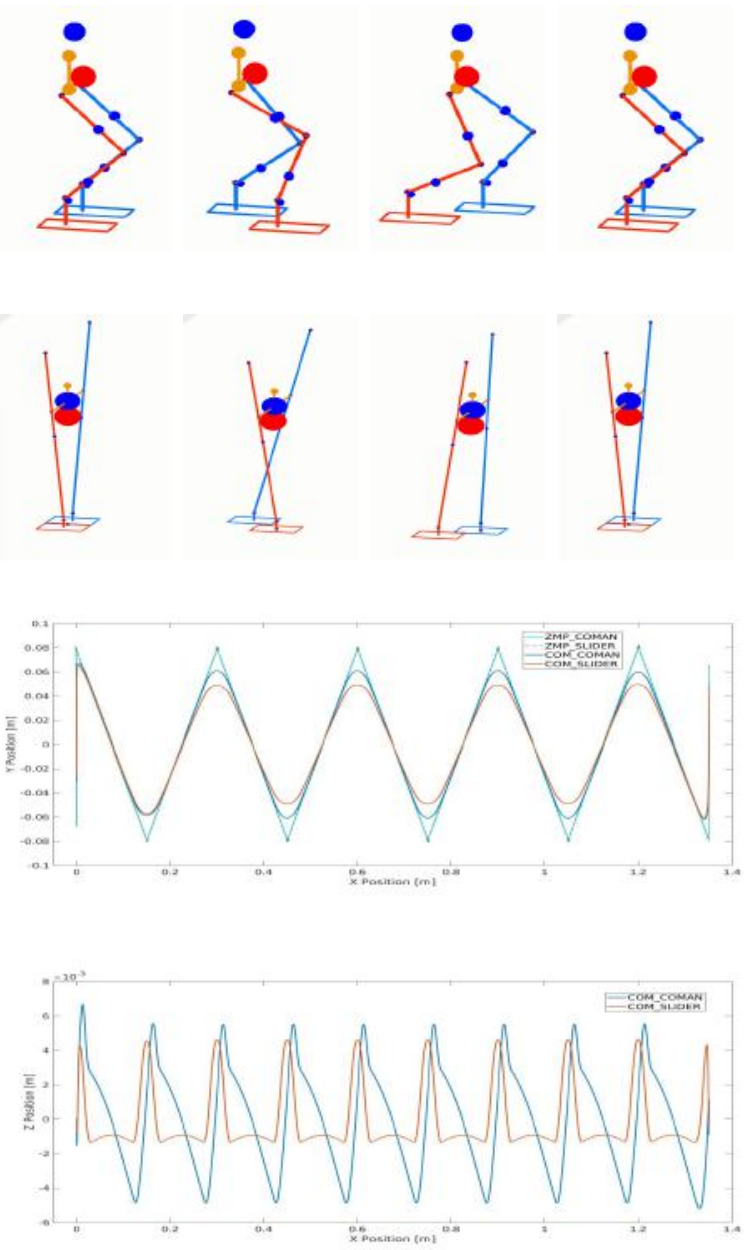

Fig. 11: Comparison of the center of mass (CoM) motion between SLIDER and COMAN with the same identical gait execution

\section{CONCLUSION AND FUTURE WORK}

The design of SLIDER, an ultra-lightweight knee-less bipedal walking robot, has been introduced. Stable walking on flat ground using a ZMP based controller was demonstrated on SLIDER, both physically and in simulation. Though acceptable for flat ground walking, more sensory feedback is required for walking on uneven terrains and push recovery. Furthermore, novel control algorithms need to be developed to demonstrate SLIDER's agility in hopping [20] and push recovery [21]. Future work will focus on mechanical enhancements as well as developments of new control algorithms.

\section{ACKNOWLEDGMENT}

We would like to thank Nemanja Rakicevic, Oscar Gill, Matt Cocking, Joseph Shore and Aksat Shah for their contribution to the conceptual, mechanical, and electronic design of the SLIDER robot. We would also like to thank Xiaobin Xiong and Aaron Ames for constructive discussions about bipedal controller design. 


\section{REFERENCES}

[1] S. Kajita, F. Kanehiro, K. Kaneko, K. Fujiwara, K. Harada, K. Yokoi, and $\mathrm{H}$. Hirukawa, "Biped walking pattern generation by using preview control of zero-moment point," in 2003 IEEE International Conference on Robotics and Automation (Cat. No. 03CH37422), vol. 2. IEEE, 2003, pp. $1620-1626$.

[2] R. J. Griffin, G. Wiedebach, S. Bertrand, A. Leonessa, and J. Pratt, "Straight-leg walking through underconstrained whole-body control," in 2018 IEEE International Conference on Robotics and Automation (ICRA). IEEE, 2018, pp. 1-5.

[3] K. Wang, A. Shah, and P. Kormushev, "SLIDER: A bipedal robot with knee-less legs and vertical hip sliding motion," in Proc. 21st International Conference on Climbing and Walking Robots and Support Technologies for Mobile Machines (CLAWAR 2018), Panama, 2018.

[4] R. R. Playter and M. H. Raibert, "Control of a biped somersault in 3d," in Proceedings of the IEEE/RSJ International Conference on Intelligent Robots and Systems, vol. 1. IEEE, 1992, pp. 582-589.

[5] S. Kajita, O. Matsumoto, and M. Saigo, "Real-time 3d walking pattern generation for a biped robot with telescopic legs," in Proceedings 2001 ICRA. IEEE International Conference on Robotics and Automation (Cat. No. 01CH37164), vol. 3. IEEE, 2001, pp. 2299-2306.

[6] Google. The schaft robot. [Online]. Available: https://www.youtube.com/watch?v=iyZE0psQsX0

[7] B. Dynamics. Atlas, the next generation. [Online]. Available: https://www.youtube.com/watch?v=fRj34o4hN4I

[8] A. Robotics. Cassie, next generation robot. [Online]. Available: https://www.youtube.com/watch?v=Is4JZqhAy-Mt=99s

[9] J. Englsberger, A. Werner, C. Ott, B. Henze, M. A. Roa, G. Garofalo, R. Burger, A. Beyer, O. Eiberger, K. Schmid et al., "Overview of the torque-controlled humanoid robot toro," in 2014 IEEE-RAS International Conference on Humanoid Robots. IEEE, 2014, pp. 916-923.

[10] B. Katz, J. Di Carlo, and S. Kim, "Mini cheetah: A platform for pushing the limits of dynamic quadruped control," in 2019 International Conference on Robotics and Automation (ICRA). IEEE, 2019, pp. 6295-6301.

[11] L. Colasanto, N. G. Tsagarakis, and D. G. Caldwell, "A compact model for the compliant humanoid robot coman," in 2012 4th IEEE RAS EMBS International Conference on Biomedical Robotics and Biomechatronics (BioRob), June 2012, pp. 688-694.

[12] "Servobelt linear drive," https://www.belleverman.com/products/linear-positioning/servobelt-linear-sblcompare, accessed: Feb 2020.

[13] J. W. Sensinger and J. H. Lipsey, "Cycloid vs. harmonic drives for use in high ratio, single stage robotic transmissions," in 2012 IEEE International Conference on Robotics and Automation. IEEE, 2012, pp. 4130-4135.

[14] A.-D. Pham and H.-J. Ahn, "High precision reducers for industrial robots driving 4th industrial revolution: state of arts, analysis, design, performance evaluation and perspective," International Journal of Precision Engineering and Manufacturing-Green Technology, vol. 5, no. 4 , pp. 519-533, 2018.

[15] Odrive. Odrive robotics. [Online]. Available: https://odriverobotics.com/

[16] O. Stasse, T. Flayols, R. Budhiraja, K. Giraud-Esclasse, J. Carpentier, J. Mirabel, A. Del Prete, P. Souères, N. Mansard, F. Lamiraux et al., "Talos: A new humanoid research platform targeted for industrial applications," in 2017 IEEE-RAS 17th International Conference on Humanoid Robotics (Humanoids). IEEE, 2017, pp. 689-695.

[17] P. Kryczka, Y. M. Shiguematsu, P. Kormushev, K. Hashimoto, H. ok Lim, and A. Takanishi, "Towards dynamically consistent realtime gait pattern generation for full-size humanoid robots," IEEE International Conference on Robotics and Biomimetics (ROBIO), 2013.

[18] S. Kajita, H. Hirukawa, K. Harada, and K. Yokoi, Introduction to Humanoid Robotics. Springer Publishing Company, Incorporated, 2014.

[19] S. Kajita, M. Morisawa, K. Miura, S. Nakaoka, K. Harada, K. Kaneko, F. Kanehiro, and K. Yokoi, "Biped walking stabilization based on linear inverted pendulum tracking," in 2010 IEEE/RSJ International Conference on Intelligent Robots and Systems. IEEE, 2010, pp. 44894496.

[20] X. Xiong and A. D. Ames, "Bipedal hopping: Reduced-order model embedding via optimization-based control," in 2018 IEEE/RSJ Inter- national Conference on Intelligent Robots and Systems (IROS). IEEE, 2018, pp. 3821-3828.

[21] S. Feng, X. Xinjilefu, C. G. Atkeson, and J. Kim, "Robust dynamic walking using online foot step optimization," in 2016 IEEE/RSJ International Conference on Intelligent Robots and Systems (IROS). IEEE, 2016, pp. 5373-5378. 